\title{
Neuropilin-1 may be responsible for retinal findings in patients with COVID-19
}

\author{
Amr Ahmed El-Arabey ${ }^{1}$ (i) $\cdot$ Mohnad Abdalla ${ }^{2}$
}

Received: 24 March 2021 / Accepted: 8 April 2021 / Published online: 13 April 2021

(c) Japan Human Cell Society 2021

Keywords COVID-19 · SARS-CoV-2 $\cdot$ NRP-1 $\cdot$ Retina $\cdot$ Hyperreflective lesions $\cdot$ VEGF $\cdot$ Cotton wool spots

Dear Editor:

Coronavirus Disease 2019 (COVID-19) caused by the SARS-CoV-2 virus has affected more than 120 million people worldwide and caused 2,672,857 deaths in 192 countries as of March 17, 2021, https://coronavirus.jhu.edu/map.html. COVID-19 can attack several body parts in different ways. Recently, Marinho et al. [1] have used non-invasive imaging optical coherence tomography to track the findings of 12 adults with COVID-19. SARS-CoV-2 infection induces hyperreflective lesions in the retinal ganglion cells of both eyes, including the papillomacular bundle. Moreover, fundus examination showed that SARS-CoV-2 triggers retinal arcade microhemorrhages and subtle cotton wool spots[1]. In this study, we would like to shed light a possible theory behind these retinal findings. Several studies reported that neuropilin-1 (NRP-1) facilitates not only the transport and cell entry into the central nervous system to develop ageusia and anosmia but also the transplacental transmission of SARS-CoV-2 [2]. Substantially, we utilized the data of single-cell analysis of retinal bipolar drop-seq [3] to examine the distribution of NRP-1 expression. Interestingly, our analysis demonstrated that NRP-1 presents in amacrine cells, Müller glia (MG), and retinal bipolar neuron cells (BC1A, $\mathrm{BC} 1 \mathrm{~B}, \mathrm{BC} 2, \mathrm{BC} 3 \mathrm{~A}, \mathrm{BC} 3 \mathrm{~B}, \mathrm{BC} 4, \mathrm{BC} 5 \mathrm{~A}, \mathrm{BC} 5 \mathrm{C}, \mathrm{BC} 5 \mathrm{D}$, and $\mathrm{BC} 8 / 9$ ) (Fig. 1). $\mathrm{MG}$ cells, the primary source for releasing inflammatory factors and cytokines in several diseases, play essential roles in detecting pathogen and host-derived ligands in retinal innate immunity. In addition, MG cells assist retinal ganglion cells with essential functions, such as the elimination of excess glutamate and the supply of energy sources [4]. Conedera et al. [5] identified the required steps for ophthalmic research to conduct a valid retinal degeneration/regeneration model. This study demonstrated that diode laser-induced hyperreflective lesions appear in the retina of zebrafish via activation of MG cells [5]. Many studies suggested that vascular endothelial growth factor (VEGF) interacts with NRP-1 to ensure the protection and functionality of the vascular system [6]. Clinically, cotton-wool spots are driven by the upregulation of VEGF [7]. Subsequently, this analysis supports the notion that COVID-19 triggers hyperreflective lesions at the retina by activating $M G$ cells via NRP-1. Furthermore, SARS-CoV-2 could promote microhemorrhages and subtle cotton-wool spots through the interaction of NRP-1 and VEGF. Future studies are required to determine the exact role of NRP-1 as a critical mediator for several retinal findings linked with SARS-CoV-2 infection in humans.

Limitations: Lack of available animal models that recapitulate the retinal findings found in SARS-CoV-2-infected humans and reliable data to test this hypothesis.

Amr Ahmed El-Arabey

ph.amrcapa@gmail.com; amrel_arabey@azhar.edu.eg

1 Department of Pharmacology and Toxicology, Faculty of Pharmacy, Al-Azhar University, Cairo, Egypt

2 Key Laboratory of Chemical Biology (Ministry of Education), Department of Pharmaceutics, School of Pharmaceutical Sciences, Cheeloo College of Medicine, Shandong University, 44 Cultural West Road, Jinan 250012, Shandong Province, People's Republic of China 


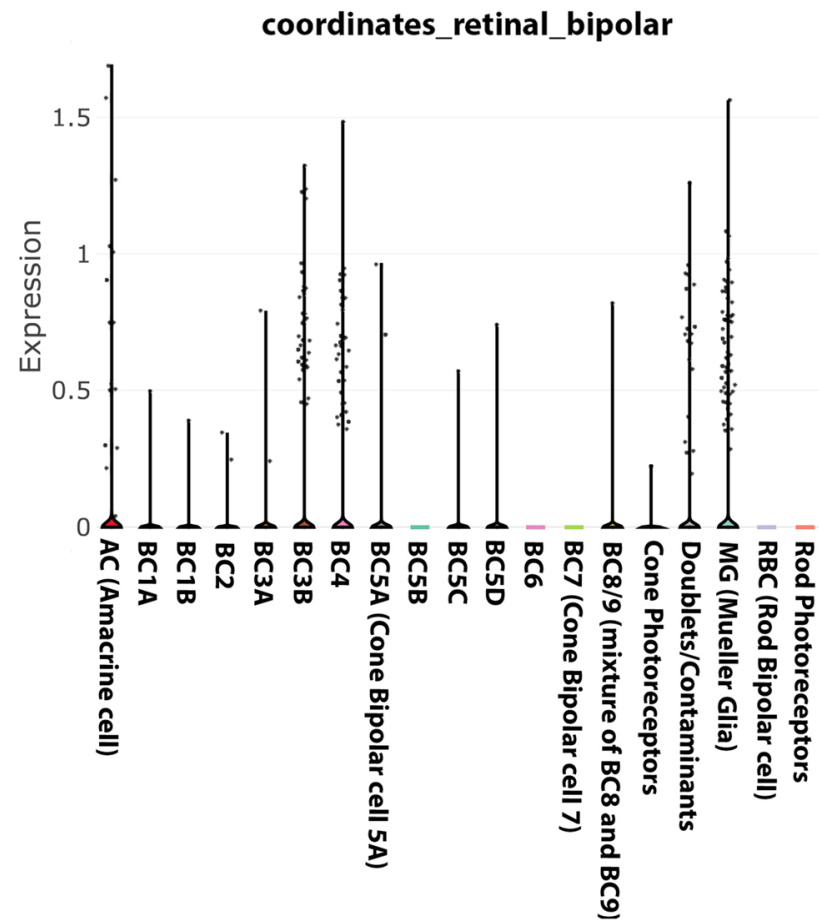

Fig. 1 Expression of NRP-1 based on single-cell transcriptomics analysis of retina

\section{References}

1. Marinho PM, Marcos AAA, Romano AC, Nascimento H, Belfort R. Retinal findings in patients with COVID-19. Lancet. 2020;395

2. El-Arabey AA, Abdalla M. Transplacental transmission of SARSCoV-2 infection via NRP1. Travel Med Infect Dis. 2021;40

3. Shekhar K, Lapan SW, Whitney IE, Tran NM, Macosko EZ, Kowalczyk M, et al. Comprehensive classification of retinal bipolar neurons by single-cell transcriptomics. Cell. 2016;166

4. Kumar A, Pandey RK, Miller LJ, Singh PK, Kanwar M. Muller glia in retinal innate immunity: a perspective on their roles in endophthalmitis. Critical Rev Immunol. 2013;33

5. Conedera FM, Arendt P, Trepp C, Tschopp M, Enzmann V. Muller glia cell activation in a laser-induced retinal degeneration and regeneration model in zebrafish. J Vis Exp. 2017; :56249

6. Mayi BS, Leibowitz JA, Woods AT, Ammon KA, Liu AE, Raja A. The role of Neuropilin-1 in COVID-19. PLOS Pathogens. 2021;17

7. Park Y-G, Jee D, Kwon J. Aqueous humor cytokine levels in diabetic macular edema patients with cotton-wool spots. J Diabetes Res. 2019;2019

Publisher's Note Springer Nature remains neutral with regard to jurisdictional claims in published maps and institutional affiliations. 